\title{
Metodología para seleccionar zonas de intervención con cultivos biofortificados
}

\author{
Fredy A. Monserrate Rojas, ${ }^{1}$ Helena Pachón, ${ }^{2}$ Glenn G. Hyman ${ }^{2}$ \\ y Andrea L. Vesga Varela ${ }^{3}$
}

Forma de citar

Monserrate Rojas FA, Pachón H, Hyman GG, Vesga Varela AL. Metodología para seleccionar zonas de intervención con cultivos biofortificados. Rev Panam Salud Publica. 2009;26(5):419-28.

RESUMEN Objetivo. Identificar zonas geográficas de América Latina y el Caribe para la biofortificación de cultivos básicos como frijol, maíz, arroz, yuca y batata, contribuyendo así a reducir las deficiencias nutricionales en la Región.

Método. Se generó un sistema de información geográfica (SIG) que incluyó registros sobre riesgos nutricionales, producción de cultivos, consumos alimenticios, y datos demográficos y socioeconómicos para 11 países de la Región. Se realizaron cuatro estudios de caso (en Guatemala, México, Bolivia y Colombia) basados en un análisis exploratorio y descriptivo de mapas temáticos, y su superposición y comparación para buscar patrones espaciales e identificar zonas candidatas de intervención.

Resultados. En Guatemala, las mayores tasas de riesgos nutricionales, producción de frijol y densidad poblacional coincidieron en las regiones Nororiental y Suroriental. En México, la distribución espacial de los niveles más altos de riesgos nutricionales, pobreza y producción de maíz se concentraron en los municipios del centro y sur. En Bolivia la producción de frijol tendió a situarse en el este del país, y el riesgo nutricional en el oeste. En Colombia, tanto los riesgos nutricionales como la producción de yuca mostraron una gran dispersión geográfica.

Conclusiones. Para Guatemala se propone la biofortificación con hierro del frijol en el sur de la región Nororiental y en la Suroriental, en México, la biofortificación del maíz con aminoácidos en los municipios productores del centro y sur del país; para Bolivia, una intervención con frijol biofortificado con hierro y zinc en zonas productoras de Santa Cruz, Chuquisaca y Tarija; y en Colombia, la biofortificación de yuca con $\beta$-caroteno en los departamentos de Córdoba y Cundinamarca.

Palabras clave Agricultura; cultivos agrícolas; deficiencia de hierro; deficiencia de zinc; desnutrición; nutrición; producción de alimentos; América Latina; Caribe.

1 Universidad Nacional de Colombia, Agronomía. Centro Internacional de Agricultura Tropical (CIAT). La correspondencia debe dirigirse a Fredy A. Monserrate Rojas, Centro Internacional de Agricultura Tropical (CIAT), Km. 17, Recta CaliPalmira, Palmira, Colombia. Correo electrónico: famonserrate@gmail.com

2 Centro Internacional de Agricultura Tropical (CIAT).

3 Universidad Industrial de Santander. Santander, Colombia.
Durante el período 2003-2005, cerca de 45 millones de personas padecieron desnutrición en América Latina y el Caribe, cifra que coloca a esta enfermedad como un importante problema de salud pública en la Región (1). En 2002 el Fondo de las Naciones Unidas para la Infancia (UNICEF), con base en estadísticas nacionales de 22 países de la Región, notificó que la prevalencia de desnutrición crónica en menores de 5 años oscilaba entre $2 \%$ en Chile y $49 \%$ en Guatemala, con valores intermedios tales como $14 \%$ en Colombia, $18 \%$ en México y $26 \%$ en Bolivia (2).

Si bien puede haber otras causas, las deficiencias nutricionales se relacionan principalmente con la calidad de la dieta 
—como sucede en la Región-y con la falta de alimentos, que es la razón más común en África. (3). De allí que en casi todos los países en desarrollo los trastornos nutricionales de mayor prevalencia incluyan malnutrición proteico-energética, carencia de vitamina A, anemias nutricionales por deficiencia de hierro, y alteraciones del crecimiento y la respuesta inmune por deficiencia de zinc (4).

Los programas para combatir las deficiencias de hierro, zinc, vitamina A y proteína, generalmente requieren tres tipos de intervenciones (5). La primera, obvia pero muchas veces difícil de implementar, consiste en diversificar la dieta mediante el aumento del consumo de carnes, frutas y verduras, las cuales tienen altos niveles de micronutrientes $y$, en el caso de la carne, de proteína completa $(4,6,7,8)$. La segunda es la distribución de suplementos dietéticos, que puede resultar efectiva para aumentar los niveles de micronutrientes en algunas poblaciones (9-11). La tercera intervención comprende la fortificación industrial de alimentos básicos, como arroz con vitaminas del complejo B y hierro, el azúcar con vitamina A y hierro, el trigo con hierro y acido fólico, y la sal con yodo $(12,13)$. Si han de ser exitosas, estas dos últimas estrategias requieren de una infraestructura apropiada que facilite su implementación.

Actualmente se encuentra en desarrollo una cuarta intervención, conocida como biofortificación, que busca aumentar el contenido nutricional y las propiedades agronómicas de determinados cultivos clave a través del fitomejoramiento convencional, para contrarrestar las deficiencias nutricionales con alimentos de alto consumo (14-19). Hay dos consorcios internacionales -AgroSalud y HarvestPlus - que trabajan en biofortificación, con el objetivo de aumentar los niveles de hierro, zinc, -caroteno y lisina y triptófano en los cultivos de frijol, maíz, batata, arroz y yuca. AgroSalud enfoca sus esfuerzos en 14 países de América Latina y el Caribe, ${ }^{4}$ mientras que el segundo lo hace en países de África y Asia. Cabe aclarar que AgroSalud utiliza la yuca biofortificada por HarvestPlus para la elaboración de productos alimenticios y también que HarvestPlus ha comenzado el proceso de

\footnotetext{
4 Bolivia, Brasil, Colombia, Costa Rica, Cuba, El Salvador, Guatemala, Haití, Honduras, México, Nicaragua, Panamá, Perú y República Dominicana.
}

biofortificación en otros cultivos básicos, como papa, trigo y cebada. Para ser efectivas, las intervenciones contra la desnutrición deben enfocar sus esfuerzos en zonas geográficas donde hay mayor impacto integrando, entre otros, datos sobre aspectos agronómicos, nutricionales, sociales y demográficos $(20,21)$. Las zonas geográficas donde la biofortificación sería más exitosa son aquellas con alto riesgo de presentar deficiencias nutricionales de hierro, zinc, vitamina A o proteína, y donde se produzcan y se consuman los cultivos que están siendo biofortificados. Igualmente, para tener mayor impacto, se busca priorizar la intervención en los sitios con mayor densidad poblacional y niveles de pobreza, siendo estos los criterios utilizados en la focalización de esfuerzos. Por esa razón, la planificación y el desarrollo de programas de biofortificación tienen necesidades únicas que los distinguen de otros tipos de intervenciones.

El presente estudio tiene como objetivo identificar zonas geográficas de América Latina y el Caribe donde la biofortificación pueda ser implementada con éxito. La metodología empleada incluyó la visualización y el análisis exploratorio de datos a nivel espacial, incluidas la descripción y superposición de mapas, y el empleo de un sistema de información geográfica $(22,23)$.

\section{MATERIALES Y MÉTODOS}

\section{Esquema metodológico}

Como punto de partida, y en base a una revisión de la literatura científica disponible, se identificaron indicadores bioquímicos y antropométricos, junto con sus respectivos puntos de corte y clasificaciones del riesgo nutricional a las deficiencias de hierro, zinc, vitamina A y proteína. Otros tipos de indicadores, como los de ingesta, fueron descartados debido a la escasa información disponible para los países objeto de estudio.

Luego se realizó la recolección y análisis de la información necesaria para elaborar mapas temáticos, a nivel subnacional, de riesgo nutricional a las deficiencias de hierro, zinc, vitamina A o proteína en los países donde opera AgroSalud. Estos instrumentos fueron complementados con mapas de producción de los cultivos de interés para el mismo consorcio (arroz, batata, frijol, maíz y yuca), mapas socioeconómicos y demo- gráficos $\mathrm{y}$, para algunos países, mapas especialmente diseñados a partir de la información existente sobre el consumo real de esos cultivos.

Finalmente los mapas elaborados se organizaron en un atlas digital a partir del cual, con la ayuda de un SIG, se realizó el análisis exploratorio de datos a nivel espacial, que incluyó la representación gráfica de la información obtenida, el análisis descriptivo de los datos, la superposición de mapas temáticos y su comparación para identificar sitios potenciales donde implementar la biofortificación de cultivos. En este estudio se muestra el análisis realizado en cuatro países (Bolivia, Colombia, Guatemala y México) de los abarcados por AgroSalud, ${ }^{5}$ escogidos por ser representativos de las situaciones que pueden presentarse al usar la metodología propuesta con diferentes combinaciones de país, nutriente y cultivo, las cuales se especifican en la sección de análisis espacial.

\section{Indicadores del riesgo nutricional}

La revisión efectuada planteó la necesidad de utilizar los indicadores de forma jerárquica: primero los bioquímicos, que miden el nivel de un nutriente en la sangre o un trastorno ocasionado por su deficiencia, utilizados para establecer riesgos nutricionales específicos en la población, y segundo los antropométricos, que indican el efecto que tiene sobre el crecimiento la deficiencia nutricional, utilizados para determinar riesgos nutricionales generales. El indicador antropométrico empleado fue el de talla baja para la edad, lo que permitió discriminar zonas con diferentes niveles de riesgo a las deficiencias nutricionales, puesto que su rango de variación estuvo entre $2 \%$ y $49 \%$. El uso de este indicador ha permitido encontrar disparidades a nivel subnacional en estudios anteriores en la Región, lo que es otro punto a favor de su utilización en este tipo de esfuerzo $(2,24)$.

Los niños menores de 5 años son uno de los dos grupos poblacionales - junto con las mujeres en edad reproductivamás vulnerables a la desnutrición. El presente estudio se basa principalmente en información sobre este grupo etario para determinar el riesgo nutricional a nivel poblacional, debido tanto a la prioridad

\footnotetext{
La información de los países restantes se encuentra disponible en la página web: www.AgroSalud.org
} 
CUADRO 1. Indicadores, puntos de corte y clasificacion del riesgo nutricional de deficiencias de hierro, zinc, vitamina A y proteínas en menores de 5 años

\begin{tabular}{|c|c|c|c|c|}
\hline \multirow[b]{2}{*}{ Descripción } & \multicolumn{4}{|c|}{ Tipo de indicador utilizado por nutriente } \\
\hline & & Bioquímicos & & Antropométricos \\
\hline Riesgo evaluado & $\begin{array}{l}\text { Deficiencia } \\
\text { de hierro }\end{array}$ & $\begin{array}{l}\text { Deficiencia } \\
\text { de zinc }\end{array}$ & $\begin{array}{c}\text { Deficiencia } \\
\text { de vitamina A }\end{array}$ & $\begin{array}{l}\text { Deficiencias de proteína, } \\
\text { hierro, zinc y vitamina A }\end{array}$ \\
\hline $\begin{array}{l}\text { Medición individuala } \\
\text { Punto de corte }\end{array}$ & $\begin{array}{l}\text { Hemoglobina } \\
<11 \mathrm{~g} / \mathrm{dL}\end{array}$ & $\begin{array}{l}\text { Zinc sérico } \\
<10 \mu \mathrm{mol} / \mathrm{L}\end{array}$ & $\begin{array}{l}\text { Retinol sérico } \\
<20 \mu \mathrm{g} / \mathrm{dL}\end{array}$ & $\begin{array}{c}\text { Talla para la edad } \\
\text { Talla para la edad }<-2 \text { puntos } Z^{c}\end{array}$ \\
\hline $\begin{array}{l}\text { Indicador de riesgo } \\
\text { poblacional }\end{array}$ & Anemia & Deficiencia & $\begin{array}{l}\text { Deficiencia } \\
\text { subclínica }\end{array}$ & Retraso del crecimiento \\
\hline $\begin{array}{l}\text { Puntos de corte } \\
\text { (según la clasificación del }^{\text {riesgo nutricional) }}{ }^{\mathrm{d}}\end{array}$ & $\begin{array}{c}<20 \text { (bajo) } \\
20-39 \text { (moderado) } \\
\geq 40 \text { (alto) }\end{array}$ & $\begin{array}{c}<20 \text { (bajo) } \\
20-39 \text { (moderado) } \\
\quad \geq 40 \text { (alto) }\end{array}$ & $\begin{array}{l}<10 \text { (bajo) } \\
10-19 \text { (moderado) } \\
\geq 20 \text { (alto) }\end{array}$ & $\begin{array}{c}<20 \text { (bajo) } \\
20-29 \text { (medio) } \\
30-39 \text { (elevado) } \\
\geq 40 \text { (muy elevado) }\end{array}$ \\
\hline
\end{tabular}

Fuente: elaborado por los autores a partir de las referencias 25-28.

a Medición realizada a cada niño menor de 5 años en la respectiva encuesta nacional.

b Por encima de ese valor se consideró que existía riesgo nutricional en cada niño menor de 5 años.

c Cada punto Z representa una desviación estándar en relación a la curva de crecimiento normal (57).

d Prevalencias a nivel poblacional para estimar el riesgo nutricional por cada nutriente.

CUADRO 2. Características de la información utilizada para los cuatro países estudiados ${ }^{a}$

\begin{tabular}{|c|c|c|c|c|c|}
\hline Variable & Característica & Guatemala & México & Bolivia & Colombia \\
\hline Nutricional & $\begin{array}{c}\text { Indicador nutricional } \\
\text { Representatividad } \\
\text { Año } \\
\text { Fuente }\end{array}$ & $\begin{array}{l}\text { Anemia } \\
\text { Regional } \\
2002 \\
(29)\end{array}$ & $\begin{array}{l}\text { Talla para la edad } \\
\text { Municipal } \\
2004 \\
\text { (32) }\end{array}$ & $\begin{array}{c}\text { Anemia y Talla para la edad } \\
\text { Departamental } \\
2003 \\
(30)\end{array}$ & $\begin{array}{c}\text { Talla para la edad } \\
\text { Departamental } \\
2005 \\
(31)\end{array}$ \\
\hline Consumo & $\begin{array}{l}\text { Representatividad } \\
\text { Año } \\
\text { Fuente }\end{array}$ & $\begin{array}{l}\text { Regional } \\
2000 \\
(40)\end{array}$ & $\begin{array}{l}- \\
-\end{array}$ & $\begin{array}{l}- \\
-\end{array}$ & $\begin{array}{l}- \\
-\end{array}$ \\
\hline Social & $\begin{array}{c}\text { Indicador de pobreza } \\
\text { Representatividad } \\
\text { Año } \\
\text { Fuente }\end{array}$ & $\begin{array}{l}\text { PEC }^{C} \\
\text { Municipal } \\
2000 \\
\text { (34) }\end{array}$ & $\begin{array}{l}\text { PE } \\
\text { Municipal } \\
2000 \\
\text { (35) }\end{array}$ & $\begin{array}{l}\mathrm{NBI}^{\mathrm{C}} \\
\text { Municipal } \\
2001 \\
(36)\end{array}$ & $\begin{array}{l}\text { NBI } \\
\text { Municipal } \\
2005 \\
\text { (37) }\end{array}$ \\
\hline
\end{tabular}

Fuente: elaborado por los autores a partir de datos incluidos en las referencias señaladas.

a Las variables de producción de cultivos (38) y densidad poblacional (33) corresponden a mapas mundiales con representatividad de $100 \mathrm{~km}^{2}$ y $1 \mathrm{~km}^{2}$, respectivamente.

${ }^{\mathrm{b}}$ En México, este indicador fue utilizado en niños escolares de 6 a 8 años. En Bolivia y Colombia, en menores de 5 años.

${ }^{\mathrm{c}} \mathrm{PE}=$ pobreza extrema; $\mathrm{NBI}=$ necesidades básicas insatisfechas.

de atención de este grupo de población, como a su susceptibilidad y la disponibilidad de información en todos los países a nivel subnacional. En el cuadro 1 se muestra un resumen de las mediciones, indicadores, puntos de corte y clasificaciones propuestas para determinar el riesgo frente a la deficiencia de cada nutriente o grupo de nutrientes (25-28).

\section{La información utilizada}

El cuadro 2 presenta un resumen de las variables e indicadores utilizados, el año de recolección, el grado de representatividad y las fuentes consultadas para los cuatro países de estudio.

El indicador de riesgo utilizado en cada caso fue determinado en función de la disponibilidad de información nutricional. Así, para la deficiencia de hierro se utilizó el indicador bioquímico de anemia en Guatemala (29) y Bolivia (30), mientras que el riesgo de sufrir deficiencias de zinc, vitamina A y proteína fue estimado mediante el indicador antropométrico de talla baja para la edad $(<-2$ desviaciones estándar [DE]) en niños menores de 5 años en Bolivia (30) y Colombia (31); y en el caso de México, en niños de entre 6 y 8 años (32).

A nivel demográfico se utilizó el mapa de densidad poblacional por $\mathrm{km}^{2}$ disponible a nivel mundial, mientras que a nivel socioeconómico fueron utilizados, para Guatemala y México, el mapa municipal de personas bajo la línea de pobreza extrema y, para Bolivia y Colombia, el porcentaje municipal de personas con necesidades básicas insatisfechas (NBI) (33-37). Adicionalmente se utilizaron mapas de producción estimada de cultivos básicos con el número de hectáreas por cada $100 \mathrm{~km}^{2}$, a nivel mundial (38), desarrollados con base en estadísticas de la Organización para la Agricultura y la Alimentación $(\mathrm{FAO})^{6}$ (39). Finalmente, para Guatemala se obtuvieron datos del consumo alimenticio real (gramos/persona/día), a nivel regional (40), el tipo de información de menor disponibilidad de las incluidas en el presente trabajo.

\section{El análisis espacial}

El punto de inicio fue un atlas diseñado en el aplicativo ArcGIS Desktop 9.1 ${ }^{\circledR}$ (41), donde se identificaron las zonas de intervención, tomando en cuenta las diferentes combinaciones de país, nutrientes y culti-

\footnotetext{
6 Información proveniente de cada país, a nivel municipal, y actualizada hasta el año 2001.
} 
vos. La técnica utilizada fue un análisis exploratorio de datos a nivel espacial (22), el cual consistió en una representación gráfica donde a cada mapa se le asignaron diferentes tonalidades según cada una de las categorías o intervalos en que fueron clasificadas las variables $(42,43)$. A continuación se procedió a describir y analizar las tendencias espaciales de los datos e identificar patrones en cada mapa y, finalmente, a realizar superposiciones y comparaciones entre los distintos mapas, estableciendo relaciones espaciales entre ellos para individualizar las zonas potenciales de biofortificación.

En los estudios de caso se presentaron las siguientes cuatro combinaciones de país, nutriente y cultivo:

- Guatemala: hierro-arroz y frijol.

- México: aminoácidos-maíz.

- Bolivia: hierro y zinc-frijol.

- Colombia: $\beta$-caroteno (vitamina A)— yuca.

Los primeros mapas analizados fueron los nutricionales, donde se identifi- caron las zonas según el riesgo de sufrir deficiencias para cada nutriente con las categorías de riesgo bajo, moderado y alto para los indicadores bioquímicos, o bajo, medio, elevado y muy elevado para los indicadores antropométricos (cuadro 1). El análisis prosiguió con los mapas relativos a la producción y el consumo del cultivo, lo que permitió determinar las zonas donde las deficiencias nutricionales pudieran ser abordadas mediante uno o varios cultivos biofortificados. Finalmente se incorporó la información socioeconómica y demográfica, identificando las zonas más vulnerables a las deficiencias nutricionales donde la intervención pudiera tener un mayor impacto.

\section{RESULTADOS}

El análisis exploratorio de la representación espacial de información fue altamente positivo puesto que permitió identificar zonas candidatas a intervenciones de forma rápida, sencilla y lógica, mediante el uso de variables con escalas tanto cualitativas como cuantitativas. A continuación se presentan los cuatro estudios de caso en los cuales se escogieron las zonas de intervención.

\section{Estudio de caso 1. Biofortificación de arroz y frijol con hierro en Guatemala}

Las variables relativas al riesgo de sufrir deficiencias de hierro y al consumo per cápita de frijol y arroz fueron desplegadas en mapas a nivel regional. Adicionalmente, se realizó el despliegue de los datos referidos a la producción de esos dos cultivos por cada $100 \mathrm{~km}^{2}$ y de las variables densidad poblacional por $\mathrm{km}^{2}$ y porcentaje municipal de personas bajo la línea de extrema pobreza rural.

En el mapa nutricional del país (figura 1, A) se observa que las regiones Nororiental, Petén, Norte y Suroriental, tienen mayor riesgo de padecer deficiencias de hierro, con un nivel de riesgo moderado y prevalencias de anemia de $36,1 \%$, $35,4 \%, 26,3 \%$ y $20,0 \%$, respectivamente, mientras que en las demás regiones el

FIGURA 1. Identificación de sitios candidatos para la biofortificación de arroz y frijol con hierro en Guatemala a nivel regional, 2009
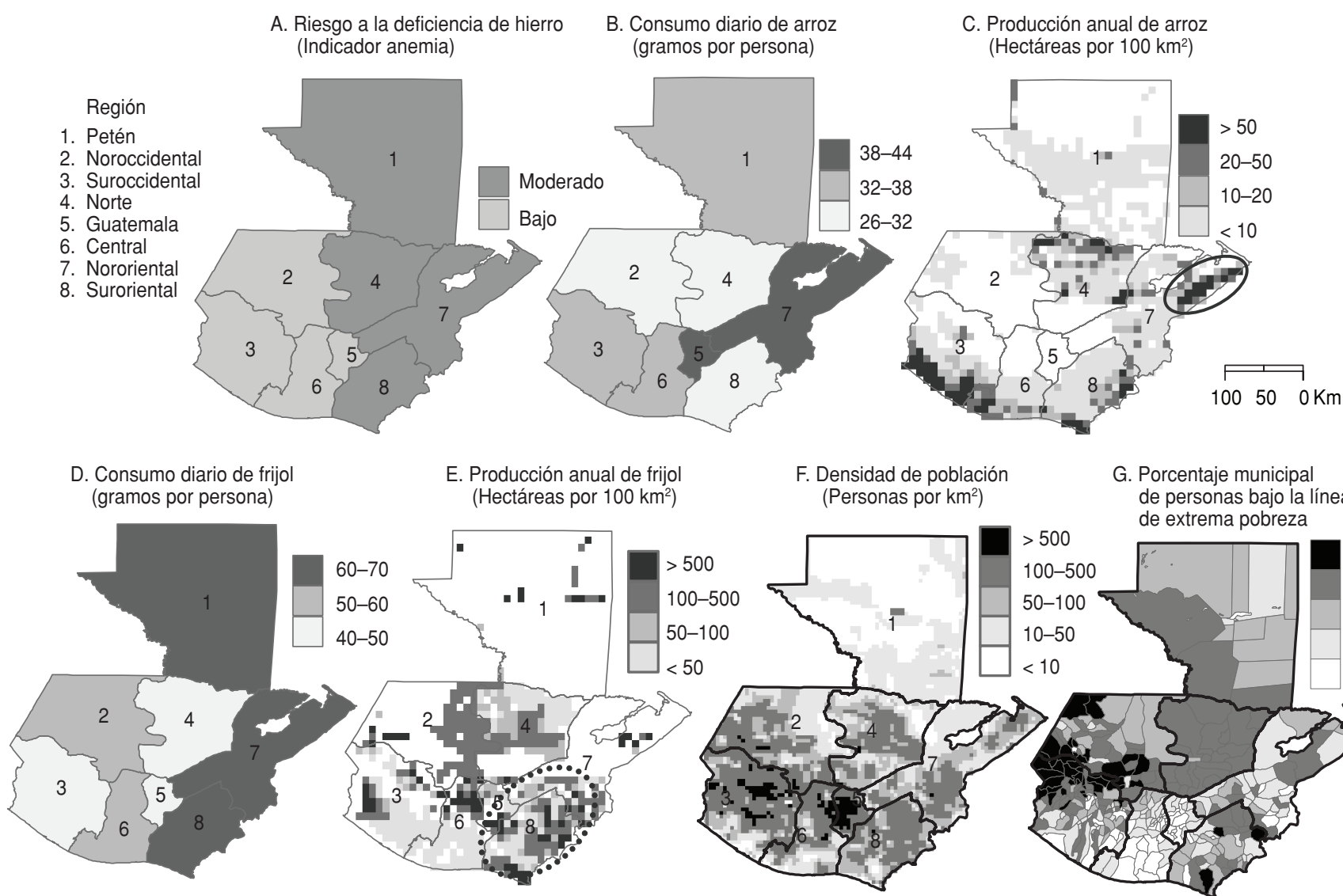

E. Producción anual de frijol
(Hectáreas por $\left.100 \mathrm{~km}^{2}\right)$

F. Densidad de población (Personas por $\mathrm{km}^{2}$ )
G. Porcentaje municipal de personas bajo la línea de extrema pobreza

Fuente: elaboración de los autores. 
riesgo es menor y se encuentra entre $1,9 \%$ en la región Suroccidental y 19,5\% en la Central.

En el caso del arroz, el consumo per cápita registró valores de entre 26 y 44 gramos por persona por día (g/persona/ día), siendo mayor en las regiones Nororiental y Guatemala, al tiempo que la distribución espacial de su producción estuvo concentrada fundamentalmente en tierras bajas y planas, localizadas en el sur hacia el Océano Pacífico y en la región Nororiental (figura 1, B y C).

Los mayores consumos de frijol se registraron en las regiones Suroriental, Petén y Nororiental con 67,1, 61,7 y $61,3 \mathrm{~g} /$ persona/día, respectivamente. La distribución espacial de la producción de esta leguminosa se encuentra localizada hacia el interior del país en tierras altas y topografías quebradas, con un patrón espacial similar a la trayectoria de la Sierra Madre (figura 1, D y E).

En relación a la densidad poblacional (figura 1, F), se aprecia un mayor número de personas por $\mathrm{km}^{2}$ en las regio- nes del centro y sur del país, en contraposición a lo que ocurre principalmente en la región de Petén, que presenta los índices más bajos. Finalmente, los porcentajes municipales de personas bajo la línea de extrema pobreza (figura 1, G) fueron mayores en las regiones Noroccidental y Suroccidental, aunque se extiende también hacia el oriente del país, especialmente en las zonas más altas.

El análisis comparado de los distintos mapas de la figura 1 permitió identificar las posibles zonas de intervención. En el caso del arroz se eligió la zona norte de la región Nororiental (figura 1, C [óvalo]), que tiene una gran concentración poblacional y dos municipios con altos porcentajes de personas bajo la línea de pobreza extrema. En el caso del frijol se eligieron las zonas sur de la región Nororiental y norte de la región Suroccidental (figura 1, E [óvalo]), que tienen gran densidad de población y municipios con altos porcentajes de personas bajo la línea de extrema pobreza rural.

\section{Estudio de caso 2. Biofortificación de maíz con aminoácidos en México}

El riesgo general de padecer deficiencias nutricionales fue establecido a nivel municipal y estatal (figura 2, A y B), y la población de estudio estuvo conformada por niños de entre 6 y 8 años, de forma distinta a los demás países. El análisis visual, a nivel estatal, mostró un riesgo nutricional bajo en todos los estados a excepción de Oaxaca, Chiapas y Yucatán, donde el riesgo fue medio, mientras que a nivel municipal se encontraron diferentes niveles de riesgo en la mayoría de los estados.

Se identificaron tres agrupaciones de municipios con riesgos nutricionales de un nivel medio, elevado y muy elevado. La primera agrupación incluyó municipios en los estados de San Luis Potosí, Hidalgo, Veracruz, Puebla, Oaxaca y Guerrero, la segunda correspondió a municipios localizados hacia la zona norte y oriente de Chiapas, y la tercera estuvo conformada por municipios de los estados de la península de Yucatán

\section{FIGURA 2. Identificación de sitios candidatos para la biofortificación de maíz con aminoácidos en México a nivel municipal y estatal, 2009}

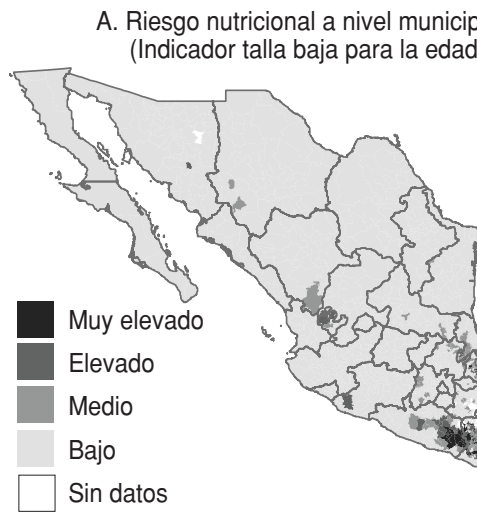

C. Producción anual de maíz (Hectáreas por $100 \mathrm{~km}^{2}$ )

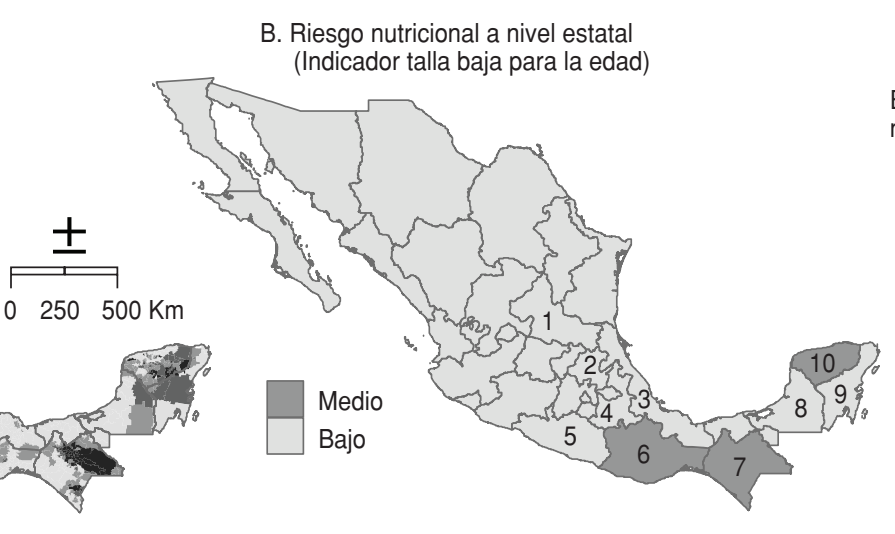

Riesgo nutricional a nivel estatal (Indicador talla baja para la edad) 
(Campeche, Quintana Roo y Yucatán). El cuadro 3 muestra un resumen de la prevalencia de talla baja para la edad a nivel estatal, junto a la menor y mayor prevalencia a nivel municipal, respecto de aquellos estados donde fueron encontradas las agrupaciones de municipios con mayores riesgos nutricionales. tuvo distribuida ampliamente desde el centro al sur del país, mostrando un patrón espacial similar al de los municipios con mayores niveles de riesgo de desnutrición (figura 2, A y C). En la figura 2 (D) se muestra el mapa con los municipios priorizados para promover la biofortificación de maíz, seleccionados en base a $\mathrm{su}$ altos riesgos nutricionales (niveles medio, elevado y muy elevado) y a la presencia de producción de maíz.
Por su parte, la producción de maíz es-

Finalmente, al incorporar en el análisis las características socioeconómicas de la población se encontró que los porcentajes de personas bajo la línea de extrema pobreza rural guardaban relación con los datos municipales de prevalencia de talla baja para la edad en niños de 6 a 8 años $\left(r^{2}=0,65 ; n=2414 ; p<0,01\right)$.

\section{Estudio de caso 3. Biofortificación de frijol con hierro y zinc en Bolivia}

El indicador de mayor jerarquía propuesto para determinar el riesgo de sufrir deficiencias de hierro es la prevalencia de anemia, la cual en este caso fue estimada a nivel departamental, en menores de 5 años. A través de ese indicador se registró un riesgo alto en todos los departamentos (prevalencia mayor al 40\%), a excepción

CUADRO 3. Prevalencias promedio de baja talla para la edad a nivel estatal, junto a la mínima y máxima a nivel municipal en los 10 estados mexicanos con mayores riesgos nutricionales

\begin{tabular}{lccc}
\hline & $\begin{array}{c}\text { Prevalencia estatal } \\
\text { Estado }\end{array}$ & \multicolumn{2}{c}{$\begin{array}{c}\text { Prevalencia municipal } \\
\text { de baja talla para la edad }\end{array}$} \\
\cline { 2 - 4 } Campeche & 11 & Mínima & Máxima \\
Chiapas & 29 & 5,8 & 32 \\
Guerrero & 20 & 2,5 & 75 \\
Hidalgo & 10 & 4,9 & 70 \\
Oaxaca & 24 & 3 & 32 \\
Puebla & 13 & 0 & 94 \\
Quintana Roo & 12 & 0 & 50 \\
San Luis Potosí & 8 & 5,8 & 38 \\
Veracruz & 11 & 0 & 28 \\
Yucatán & 20 & 0 & 85 \\
\hline Funde: la edad & 0 & 65
\end{tabular}

Fuente: elaborado por los autores a partir de datos incluidos en el II Censo nacional CENTALLA, México DF (32). de Santa Cruz y Tarija donde se registraron riesgos moderados y prevalencias de $39,8 \%$ y $38,7 \%$, respectivamente.

El paso siguiente fue utilizar el indicador antropométrico de talla baja para la edad en menores de 5 años, a nivel departamental (figura 3, A), con objeto de determinar el riesgo nutricional de sufrir deficiencias de hierro y zinc. Si bien los departamentos del occidente tuvieron un mayor riesgo que los del oriente, ninguno presentó un riesgo bajo. Así, los departamentos de La Paz, Cochabamba y Oruro, ubicados al occidente, registraron riesgos nutricionales muy elevados con prevalencias de talla baja para la edad de $46 \%, 41,8 \%$ y $42,4 \%$, respectivamente. Los departamentos de Chuquisaca, Potosí y Beni/Pando, por su parte, tuvieron un riesgo nutricional elevado con prevalencias de $36,4 \%, 35,6 \%$ y 33,4\%, respectivamente. Por último, los departamentos de Santa Cruz y Tarija registraron un riesgo nutricional medio, con prevalencias de talla baja para la edad de 21,9\% y $20,6 \%$, respectivamente.

En Bolivia la distribución espacial de la producción de frijol (figura 3, B) se encuentra concentrada en el departamento de Santa Cruz y se extiende hacia el sur en la zona central de los departamentos de Chuquisaca y Tarija. Por otro lado, los mayores porcentajes municipales de personas con NBI (figura 3, C) siguen el mismo patrón observado para el riesgo nutricional, concentrándose hacia el occidente del país.

\section{FIGURA 3. Identificación de sitios candidatos para la biofortificación de frijol con hierro y zinc en los departamentos de Bolivia, 2009}

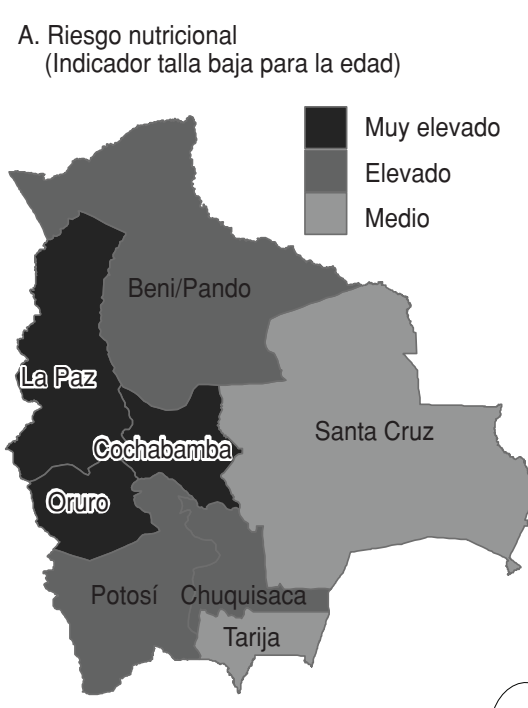

Fuente: elaboración de los autores.

\section{B. Producción anual de frijol} (Hectáreas por $100 \mathrm{~km}^{2}$ )

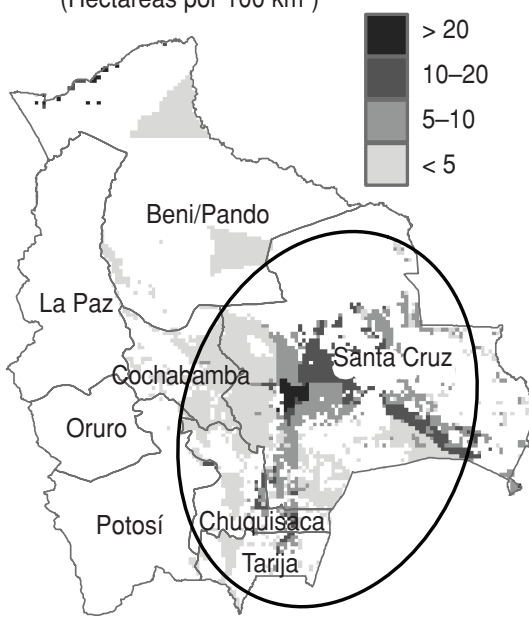

Zonas priorizadas para la intervención con variedades biofortificadas con Fe y $\mathrm{Zn}$
C. Porcentaje municipal de personas con necesidades básicas insatisfechas

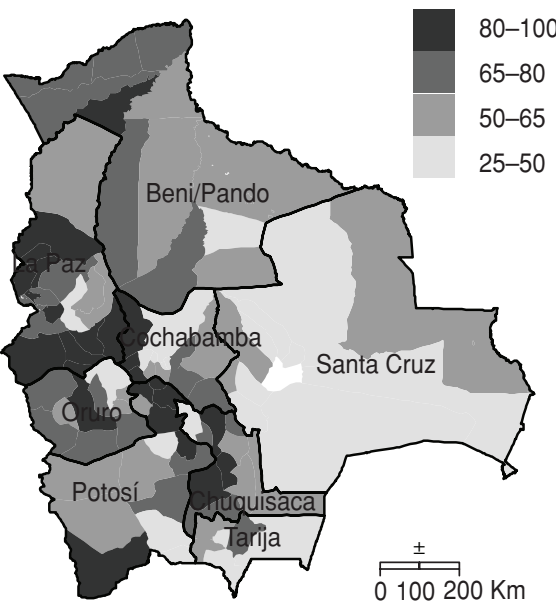


FIGURA 4. Identificación de sitios candidatos para la biofortificación de yuca con $\beta$-caroteno en Colombia a nivel departamental, 2009

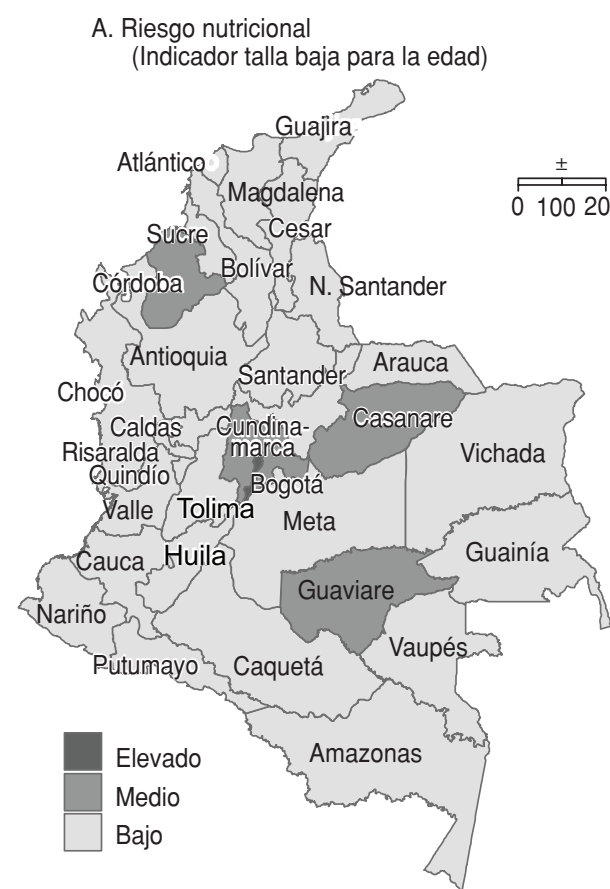

Fuente: elaboración de los autores.

Como consecuencia de lo expuesto, se planteó la necesidad de dar prioridad a la intervención en los departamentos de Santa Cruz, Tarija y Chuquisaca (figura 3, B [óvalo]).

\section{Estudio de caso 4. Biofortificación de yuca con $\beta$-caroteno en Colombia}

El indicador de mayor jerarquía propuesto para establecer el riesgo a la deficiencia de vitamina A fue la prevalencia de deficiencia subclínica de vitamina A (DSVA), determinada con base en los niveles de retinol sérico en menores de 5 años (31) (cuadro 1). El indicador fue notificado únicamente para la región atlántica (Guajira, Magdalena, Atlántico, Cesar, Bolívar, Sucre y Córdoba), donde la DSVA alcanzó $14 \%$ con un riesgo moderado, razón por la cual en otras regiones se utilizó el indicador antropométrico de talla baja para la edad a nivel departamental (figura 4, A), por ser el siguiente en la jerarquía propuesta para definir el riesgo nutricional. En la figura 4 se puede observar un riesgo nutricional elevado en Bogotá, con prevalencia de talla baja para la edad de $31,5 \%$, y un riesgo medio en los departamentos de Guaviare, Córdoba, Casanare y Cundinamarca, con prevalencias de $27,1 \%$, $25,9 \%, 22,1 \%$ y $20,3 \%$, respectivamente. En los demás departamentos se encontró
B. Producción anual de yuca (Hectáreas por 100 km²)

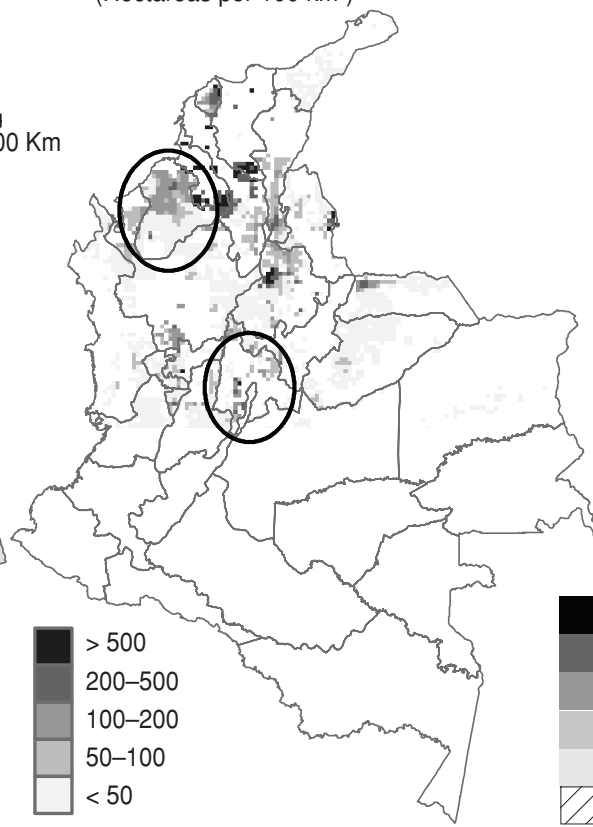

$<50$
C. Porcentaje municipal de personas con necesidades básicas insatisfechas

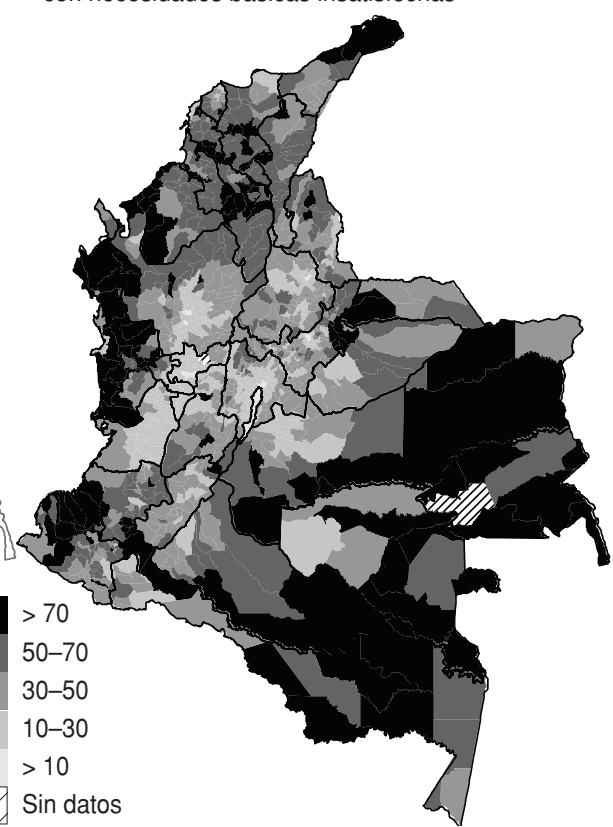

un riesgo nutricional bajo, con prevalencias menores a $20 \%$.

El análisis de la distribución espacial de la producción de yuca en Colombia permitió identificar cuatro zonas productoras principales (figura 4, B). La primera, localizada en la región atlántica, comprende los departamentos Atlántico, Magdalena, Córdoba, Sucre y Cesar, extendiéndose hacia el sur en el departamento de Santander. La segunda fue identificada en la región cafetera, al sur del departamento de Antioquia y en los departamentos de Caldas, Risaralda y Quindío. La tercera zona corresponde a los departamentos del Tolima, Huila, parte de Cundinamarca y nororiente del Caquetá y la cuarta se encuentra en la región de los Llanos Orientales, en los departamentos de Arauca y Casanare.

En términos de consumo de yuca en el país, se ha documentado un promedio anual por persona que puede alcanzar $41,1 \mathrm{~kg}$ y $17,1 \mathrm{~kg}$ en regiones rurales y urbanas respectivamente, dándose los mayores consumos en las zonas productoras y sus alrededores (44). Por esa razón la biofortificación de yuca con $\beta$-caroteno puede ser una alternativa eficaz para combatir la deficiencia nutricional de vitamina A, principalmente en las zonas de producción de Córdoba (región Atlántica), y en los departamentos de Casanare y Cundinamarca (figura 4, B [óvalos]).
Adicionalmente, al analizar la distribución espacial de personas con NBI en los municipios (figura 4,C), se observó que los departamentos priorizados incluían municipios con índices de NBI mayores a $70 \%$, en especial al sur del departamento de Córdoba. Sin embargo, llamó la atención el bajo riesgo nutricional registrado en el departamento de Chocó (prevalencia de talla baja para la edad en niños, $16,8 \%$ ), donde $77,4 \%$ de los municipios mostraban índices de NBI mayores a $50 \%$. La situación opuesta se observó en Bogotá, donde con el mayor riesgo nutricional del país se obtuvieron índices de NBI de 9,2\%.

\section{DISCUSIÓN}

Si bien dentro de un SIG es posible utilizar una variedad de herramientas de análisis y modelamiento, como el álgebra de mapas, el análisis multicriterio y la superposición ponderada (45-48), un primer paso posible -y muy efectivoes el análisis exploratorio de datos, que permite al investigador identificar tendencias en las distintas variables y establecer relaciones entre ellas. El hecho de haber determinado con precisión los criterios de selección de zonas candidatas para la biofortificación, y de haber seguido los pasos del proceso expuesto, posibilitó la rápida identificación de esas 
zonas potenciales. Asimismo, se consideró pertinente dar a conocer los resultados a las personas que intervienen en programas de biofortificación en la Región (fitomejoradores, distribuidores de semilla, nutricionistas).

La mayor profundidad en el manejo de la información y la utilización de técnicas diferentes a las descriptivas, como la superposición ponderada de mapas (49), han refinado la selección de zonas potenciales haciendo posible corroborar los resultados presentados.

En el caso de Guatemala, las zonas de intervención en los cultivos de arroz y frijol no resultaron ser las mismas debido a variables agroecológicas que definen el rango de adaptación de cada cultivo. Por tal razón, es importante tener en cuenta los rangos de adaptación de los cultivos y de las diferentes variedades biofortificadas.

Los resultados del análisis del consumo de arroz y frijol en Guatemala plantean la necesidad de priorizar la intervención con variedades de frijol, debido al bajo consumo de arroz en el país comparado con el promedio de América Latina y el Caribe (83 g/persona/día) (50). A una conclusión similar se arribó al consultar con los fitomejoradores, cuando quedó claro que la ausencia de programas nacionales de mejoramiento y distribución de semillas, y el bajo consumo de arroz, podrían dificultar esa intervención, a diferencia de lo que sucede con el frijol. En este último caso, los esfuerzos podrían dirigirse hacia una alianza con socios estratégicos que pudieran realizar la evaluación y distribución de semillas biofortificadas. Es importante agregar que una intervención en las regiones Nororiental y Suroriental - señaladas como prioritarias en el caso del frijol- podría llegar a un mayor número de personas que otra realizada en la región de Petén. Finalmente, en las zonas priorizadas para la biofortificación con frijol, existe un mayor número de municipios con altos porcentajes de personas bajo la línea de extrema pobreza, en comparación con las zonas potenciales para la biofortificación con arroz.

En México existe una larga tradición de consumo de maíz en prácticamente en toda la población, por lo que es recomendable una intervención con variedades de maíz de alta calidad de proteína QPM, con mayor contenido de los aminoácidos esenciales triptófano y lisina $(51,52)$. Esa intervención estaría orien- tada a personas con mayores riesgos de sufrir deficiencias nutricionales, debido a la coincidencia entre los patrones espaciales de la pobreza y las zonas de producción de maíz, como se ha notificado en estudios anteriores (51). La mayor preocupación, no obstante, fue el acceso de los agricultores de bajos recursos a las semillas QPM y a los cultivos biofortificados en general, situación tenida en cuenta en el desarrollo de la estrategia (53), en la que se buscó aumentar las tasas de adopción de nuevas variedades, favoreciendo así la biofortificación.

A nivel geográfico y con base en el caso de México, al momento de representar el riesgo nutricional en los niveles municipal y estatal, se puso en evidencia una limitación en el análisis de la información de fenómenos sociales, en virtud de la cual mientras se observaba una variable homogénea dentro de una región, existían variaciones extremas ocultas (2). Esa situación puede generar un fenómeno conocido como "falacia ecológica", por la cual se arriba a conclusiones sobre un nivel particular (p.ej. la prevalencia de talla baja para la edad en un municipio) a partir de un nivel general (p.ej. la prevalencia de talla baja para la edad en el estado) $(54,55)$. Por ese motivo es sumamente necesario respetar el nivel de representatividad de los datos al momento de priorizar las zonas de intervención.

En el caso de Bolivia, las zonas de intervención propuestas (departamentos de Santa Cruz, Chuquisaca y Tarija) difieren de las que poseen las mayores tasas de riesgo nutricional y de personas con NBI, debido a la necesidad de priorizar lugares donde se producen los cultivos estudiados. A propósito hay que señalar que las zonas aquí indicadas también presentan riesgos nutricionales que ameritan una intervención con cultivos biofortificados. Otros argumentos que pesaron a la hora de seleccionar las zonas de intervención incluyeron las altas tasas de adopción de nuevas variedades (56) y las características particulares de la actividad agrícola del departamento de Santa Cruz, que cuenta con los mayores niveles de producción de frijol y otros cultivos como arroz, batata, maíz y yuca (38). Tales niveles de producción determinan que ese departamento provea muchos de los alimentos que se consumen en otras regiones, tanto en Bolivia como en zonas urbanas del oriente de Brasil. Esto abre la posibilidad de producir cultivos biofortificados en un departamento productor y extender los beneficios hacia otro departamento consumidor, alternativa que ha sido soslayada en este estudio.

$\mathrm{Al}$ analizar la producción de frijol en Santa Cruz se encontró que, entre los años 1990 y 1999, la cantidad de tierra destinada a tal fin aumentó de 7000 a 28000 hectáreas, fenómeno que estuvo acompañado de una migración de personas desde los Andes hacia ese departamento, así como de una mejora en su calidad de vida (56). Por su parte, en el caso de los departamentos de Chuquisaca y Tarija, la intervención en la zona propuesta sería capaz de atender problemas nutricionales de poblaciones con tasas altas de NBI.

En Colombia, por último, se observa que las mayores tasas de riesgo nutricional y de personas con NBI no siempre tienen una distribución espacial concordante, lo que parece indicar que las comunidades con riesgos nutricionales más importantes en ocasiones no están localizadas en las zonas con mayores niveles de pobreza, de modo similar a lo observado en el occidente de Guatemala (figuras 1 y 4). Los ejemplos más visibles de esa situación incluyen al departamento de Chocó, con bajo nivel de riesgo nutricional y alto porcentaje de personas con NBI, y a la capital Bogotá, donde se da la ecuación opuesta. Por esa razón, es importante utilizar indicadores de carácter nutricional específico para determinar las poblaciones en riesgo, y encontrar otros sensibles a riesgos nutricionales específicos.

\section{Conclusiones y recomendaciones}

Desde el punto de vista metodológico, y dado que la biofortificación es una estrategia basada en la producción de cultivos, la priorización de las zonas de intervención debe realizarse teniendo en cuenta variables clave tales como zonas de producción, rangos de adaptación, infraestructura para el fitomejoramiento, distribución de semillas y adopción de variedades.

Aun cuando el estudio estuvo centrado en las zonas de producción, es obvio que el beneficio de los cultivos biofortificados se extendería a los lugares donde esos cultivos son consumidos, que pueden o no coincidir con los de producción. En relación a los cuatro países donde se efectuaron los estudios de caso, se proponen las siguientes intervenciones: 
- Guatemala: la biofortificación con hierro de frijol en el sur de la región Nororiental y en la región Suroriental (figura 1, E [ovalo]).

- México: la biofortificación del maíz con aminoácidos en los municipios productores del centro y sur del país que presentaron algún nivel de riesgo nutricional (figura 2, D).

- Bolivia: la biofortificación del frijol con hierro y zinc en las zonas productoras de los departamentos de Santa Cruz, Chuquisaca y Tarija (figura 3, B [óvalo]).

- Colombia: la biofortificación de la yuca con $\beta$-caroteno en los departamentos de Córdoba y Cundinamarca (figura 4, B [óvalos]).
En el análisis exploratorio a nivel espacial realizado para definir las zonas de intervención prioritarias, se hizo evidente una gran heterogeneidad en términos de combinaciones de factores tales como grados de riesgo nutricional, zonas de producción, zonas de consumo y zonas de mayor pobreza. De allí que al momento de definir las zonas, las variables que aportaron en mayor medida a la decisión resultaron ser diferentes en cada país, por lo cual se consideró que la utilización de un modelo rígido para todos los países no resultaría apropiada.

La principal dificultad enfrentada en el presente trabajo fue la disponibilidad y representatividad de la información, razón por la cual tanto el análisis como las con-

\section{REFERENCIAS}

1. Organización de las Naciones Unidas para la Agricultura y la Alimentación (FAO). El estado de la inseguridad alimentaria en el mundo, 2008. Hallado en: http://www.fao. org/docrep/011/i0291s/i0291s00.htm. Acceso el 29 de septiembre de 2009.

2. Kamatsuchi M. Las disparidades en América Latina y el Caribe: Evaluación rápida de la situación de nutrición. UNICEF, Sección de Nutrición, Oficina Regional para América Latina y el Caribe; 2003. Pp. 14. Hallado en: www. unicef.org/lac/disparidades.doc. Acceso el 2 de septiembre de 2009.

3. Victora $\mathrm{C}$. The association between wasting and stunting: an international perspective. J Nutr. 1992;112(5):1105-11.

4. Latham MC. Human nutrition in the developing world. Food and Nutrition Series No. 29. Roma: FAO; 1997. Hallado en: http://www. fao.org/docrep/W0073E/w0073E00.HTM. Acceso el 2 de septiembre de 2009.

5. Allen LH. Interventions for micronutrient deficiency control in developing countries: past, present and future. J Nutr. 2003;133(11S-II): 3875.

6. Gibson RS, Hotz C. Dietary diversification/ modification strategies to enhance micronutrient content and bioavailability of diets in developing countries. Br J Nutr. 2001;85(supl 2):S159-66.

7. Gibson RS, Yeudall F, Drost N, Mtitimuni BM, Cullinan TR. Experiences of a communitybased dietary intervention to enhance micronutrient adequacy of diets low in animal source foods and high in phytate: a case study in rural Malawian children. J Nutr. 2003; 133(11 supl 2):3992S-9S

8. International Zinc Nutrition Consultative Group. Assessment of the risk of zinc deficiency in populations and options for its control. Food Nutr Bull. 2004;25:S91-204.

9. Pan American Health Organization. Providing vitamin A supplements through immunization and maternal and child health contacts for children 6-24 months and mothers up to 6 weeks postpartum: A guide for health workers. Washington DC: PAHO; 2001. Pp. 12.
Hallado en: http://www.paho.org/English/ $\mathrm{HPP} / \mathrm{HPN} /$ VitAManual2ed.pdf. Acceso el 2 de septiembre de 2009.

10. Yip R. Iron Supplementation: Country Level Experiences and Lessons Learned. J Nutr. 2002;132:859S-61S

11. Shrimpton R, Gross R, Darnton-Hill I, Young $M$. Zinc deficiency: what are the most appropriate interventions? Br Med J. 2005;330: 347-9.

12. Lotfi M, Venkatesh M, Merx R, Naber-van P. Micronutrient fortification of foods: current practices, research, and opportunities. Ottawa: Micronutrient Initiative; 1996. Pp. 108

13. Brown KH, Lopez D, Arsenault JE, Peerson JM, Penny ME. Comparison of the effects of zinc delivered in a fortified food or a liquid supplement on the growth, morbidity, and plasma zinc concentrations of young Peruvian children 1-3 years. Am J Clin Nutr. 2007;85:538-47.

14. Bouis $\mathrm{H}$. Economics of enhanced micronutrient density in food staples. Field Crops Res. 1999;(60):165-73.

15. Welch R, Graham RD. A new paradigm for world agriculture: meeting human needsproductive, sustainable, nutritious. Field Crops Res. 1999;60(1-2):1-10.

16. Welch R. Breeding strategies for biofortified staple plant foods to reduce micronutrient malnutrition globally, symposium plant breeding: A new tool for fighting micronutrient malnutrition. J Nutr. 2002;132:495S-9S.

17. Welch R, Graham RD. Breeding for micronutrients in staple food crops from a human nutrition perspective. J Exp Bot. 2004;55(396): 353-64.

18. Nestel P, Bouis H, Meenakshi J, Pfeiffer W. Biofortification of Staple Food Crops. J Nutr. 2006;136:1064-7.

19. Johns T, Eyzaguirre PB. Biofortification, biodiversity and diet: A search for complementary applications against poverty and malnutrition. Food Policy. 2007;32:1-24.

20. Margai FM. Geographic Targeting of Risk Zones for Childhood Stunting and Related Health Outcomes in Burkina Faso. World Health Popul. 2007; June:1-19. clusiones debieron limitarse al marco de los datos disponibles utilizados. En el futuro se recomienda emplear indicadores nutricionales específicos y de alta sensibilidad, así como información confiable referida al consumo de nutrientes.

El uso de técnicas tales como la superposición de mapas, la representación gráfica y el análisis visual de mapas de diferentes temáticas permitió identificar con mayor exactitud zonas candidatas para la intervención en los cuatro países analizados. En la actualidad, las técnicas descritas - junto a algunas otras- constituyen un aporte significativo para priorizar esfuerzos en los 14 países con programas de biofortificación para los cuales se recolectó información.
21. Morris SS, Flores R, Zuniga M. Geographic targeting of nutrition programs can substantially affect the severity of stunting in Honduras. J Nutr. 2000;130(10):2514-9.

22. Haining R, Wise S, Ma J. Exploratory Spatial Data Analysis. J R Stat Soc Ser C Appl Stat. 1998;47(3):457-69.

23. Rushton G. Public Health, Gis, and Spatial Analytic Tools. Annu Rev Public Health. 2003;24:43-56.

24. Frongillo EA, Onis MD, Hanson KMP. Socioeconomic and Demographic Factors Are Associated with Worldwide Patterns of Stunting and Wasting of Children. J Nutr. 1997; 127(12):2302-9.

25. Center for Disease Control and Prevention (CDC). Recommendations to Prevent and Control Iron Deficiency in the United States. Morbidity and Mortality Weekly Report. 1998;47(RR-3):1-36.

26. Mora JO, Gueri M, Mora OL. Vitamin A deficiency in Latin America and the Caribbean: An Overview. Pan Am J Public Health. 1998; 4(3):178-86.

27. International Zinc Nutrition Consultative Group (IZiNCG). Assessment of the Risk of Zinc Deficiency in Populations and Options for its Control. Food and Nutr Bull. 2004;25: S130-2.

28. Maire B, Delpeuch F. Indicadores de nutrición para el desarrollo: Guía de referencia, 2006. Hallado en: ftp://ftp.fao.org/docrep/fao/ 009/y5773s/y5773s.pdf. Acceso el 2 de septiembre de 2009.

29. Instituto de Estadística de Guatemala (INE). Encuesta nacional de salud materno infantil, 2002. Hallado en: http://encuestas.ccp.ucr.ac. $\mathrm{cr} /$ camerica/gu02.html. Acceso el 2 de septiembre de 2009.

30. Instituto Nacional de Estadística (INE) de Bolivia. Encuesta nacional de demografía y salud, 2003. Hallado en: http://www.measuredhs. com/countries/. Acceso el 2 de septiembre de 2009.

31. Instituto Colombiano de Bienestar Familiar. Encuesta nacional de la situación nutricional en Colombia, 2005. Pp. 446. Hallado en: http:// 
www.icbf.gov.co/icbf/directorio/portel/ libreria/pdf/1ENSINLIBROCOM PLETO.pdf. Acceso el 28 de septiembre de 2009.

32. Instituto Nacional de Ciencias Médicas y Nutrición Salvador Zubirán (INNSZ). II Censo nacional de talla "CENTALLA". México D.F., 2004.

33. Center for International Earth Science Information Network (CIESIN), Columbia University, Centro Internacional de Agricultura Tropical (CIAT). Gridded Population of the World (GPW). 3 ed. Nueva York: Palisades; 2005. Hallado en: http://sedac.ciesin.columbia.edu/ gpw/index.jsp. Acceso el 23 de mayo de 2009.

34. Asociación de Investigación y Estudios Sociales (ASIES). Mapas de pobreza y desigualdad de Guatemala. 2005. Pp. 45. Hallado en: http:// www.ciesin.columbia.edu/repository/pov map/methods/Mapas_de_la_Pobreza_2002. pdf. Acceso el 29 de septiembre de 2009.

35. Instituto Nacional de Estadística, Geografía e Informática (INEGI), Centro Latinoamericano y Caribeño de Demografía (CELADE). Censo de población y vivienda, 2000. Aguascalientes, México.

36. Unidad de Análisis de Políticas Sociales y Económicas (UDAPE). Instituto Nacional de Estadísticas (INE) y Banco Mundial. Encuesta continua de hogares, 2001. La Paz, Bolivia.

37. Departamento Administrativo Nacional de Estadística (DANE). Censo general de población y vivienda, 2005. Hallado en: http://www. dane.gov.co/censo/. Acceso el 2 de septiembre de 2009.

38. You L, Wood S. An entropy approach to spatial disaggregation of agricultural production. Agric Syst. 2006;90:329-47.

39. Food and Agriculture Organization of the United Nations (FAO). Agro-MAPS: A global spatial database of agricultural land-use statistics aggregated by sub-national administrative districts, 2001. Hallado en: http://www. fao. org/landandwater/agll/agromaps/inter active/page.jspx. Acceso el 23 de mayo de 2009.

40. Instituto Nacional de Estadística (INE) de Guatemala. Encuesta Nacional sobre Condiciones de Vida (ENCOVI), 2000. Hallado en: http:/ / econ.worldbank.org/WBSITE/ EXTERNAL/EXTDEC/EXTRESEARCH/EX TLSMS / 0,,contentMDK:21485765 pagePK: 64168445 piPK:64168309 theSitePK: 3358997,00.html. Acceso el 23 de mayo de 2009.

41. Environmental System Research Institute (ESRI). ArcGIS Desktop Help. Redlands, California, USA. 2005. Hallado en: http://web help.esri.com/arcgisdesktop/9.1/index.cfm? TopicName=welcome. Acceso el 29 de septiembre de 200942. Unwin D. GIS, spatial analysis and spatial statistics. Prog Hum Geogr. 1996;20(4):540-51.

43. Brewer C. Designing better maps: A guide for GIS Users. California: ESRI Press; 2005. P. 203.

44. Gottret M, Escobar Z, Pérez S. El sector yuquero en Colombia: desarrollo y competitividad. En: Ospina B, Ceballos H, eds. La yuca en el tercer milenio: sistemas modernos de producción, procesamiento, utilización y comercialización. Cali: CIAT; 2002. Pp. 340-76.

45. Burrough PA, McDonnell RA. Principles of geographical information systems. Oxford: Oxford University Press; 1998. P. 327.

46. Chakhar S, Mousseau V. An algebra for multicriteria spatial modeling. Comput Environ Urban Syst. 2007;31(5):572-96.

47. Carver $\mathrm{S}$. Integrating multi-criteria evaluation with geographical information systems. Int J Geogr Inf Sci. 1991;5(3):321-39.

48. Li X, Wang W, Li F, Deng X. GIS based map overlay method for comprehensive assessment of road environmental impact. Transportation Research Part D: Transport and Environment. 1999;4(3):147-58.
49. Zapata-Caldas E, Hyman G, Pachón H, Monserrate $\mathrm{F}$, Vesga L. Identifying candidate sites for crop biofortification in Latin America: case studies in Colombia, Nicaragua and Bolivia. Int J Health Geogr. 2009:8(29).

50. Martínez C, Borrero J, Tohme J. Variedades de arroz con mayor valor nutricional para combatir la desnutrición en América Latina. Segundo Congreso Arrocero. San José, Costa Rica: CONARROZ; 2006. P. 8.

51. Bellon M, Hodson D, Bergvinson D, Beck D, Martinez-Romero E, Montoya Y. Targeting agricultural research to benefit poor farmers: Relating poverty mapping to maize environments in México. Food Policy. 2005;30(5-6): 476-92.

52. Krivanek AF, De Groote H, Gunaratna NS, Diallo AO, Friesen D. Breeding and disseminating quality protein maize (QPM) for Africa. Afr J Biotechnol. 2007;6(4):312-24.

53. Córdova H. Quality Protein Maize: Improved Nutrition and Livelihoods for the Poor. México D.F.: CIMMYT; 2000. Pp. 27-31.

54. Freedman D. Ecological inference and the ecological fallacy. International Encyclopedia of the Social and Behavioral Sciences. 1999;6: 4027-30.

55. Robinson W. Ecological correlations and the behavior of individuals. Am Sociol Rev. 1950; 15:351-7.

56. Voysest Voysest O. Creyeron en el frijol: Antiguos mineros bolivianos transforman en oro un grano alimenticio. Cultivando Afinidades. 1999. Pp. 1, 6-7.

Manuscrito recibido el 9 de febrero de 2009. Aceptado para publicación, tras revisión, el 20 de junio de 2009.

ABSTRACT Objective. To identify geographical areas in Latin America and the Caribbean where biofortification of staple crops, such as beans, corn, rice, cassava, and sweet potatoes, might help reduce nutritional deficiencies in the Region.

\section{Methodology for selecting areas for biofortified crop intervention}

Key words

Methods. A geographic information system (GIS) was produced with records on nutritional risks, crop production, food consumption, and demographic and socioeconomic data, for 11 countries in the Region. Four case studies were conducted (in Bolivia, Colombia, Guatemala, and Mexico) using exploratory and descriptive analysis of thematic maps that were superimposed and compared to reveal overlapping and spatial patterns, thereby identifying areas suited to intervention.

Results. In Guatemala, the highest rates of nutritional risk, bean production, and population density overlapped in the northeast and southeast areas. In Mexico, spatial distribution of the highest risk levels for nutrition, poverty, and corn production were concentrated in the central and southern municipalities. In Bolivia, bean production tended to be in the eastern part of the country, and nutritional risk, in the west. In Colombia, both nutritional risk and cassava production showed wide geographic dispersion.

Conclusions. For Guatemala, we propose iron biofortification of beans in the southern parts of the northeast and southeast; for Mexico, amino-acid biofortification of corn in the central and southern municipalities that produce it; for Bolivia, iron and zinc biofortification of beans in the bean-producing areas of Santa Cruz, Chuquisaca, and Tarija; and for Colombia, $\beta$-carotene biofortification of cassava in the Cordoba and Cundinamarca departments.

Agriculture; crops; iron deficiency; zinc deficiency; malnutrition; nutrition; food production; Latin America; Caribbean Region. 